\title{
LES DÉFIS DE FINS DE CARRIÈRE ET LA RETRAITE : LE CAS DU QUÉBEC
}

\author{
Diane-Gabrielle Tremblay et Maryse Larivière
}

Management Prospective Ed. | Management \& Avenir

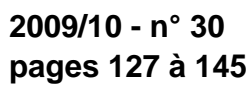

ISSN 1768-5958

Article disponible en ligne à l'adresse:

http://www.cairn.info/revue-management-et-avenir-2009-10-page-127.htm

Pour citer cet article :

Tremblay Diane-Gabrielle et Larivière Maryse, « Les défis de fins de carrière et la retraite : le cas du Québec », Management \& Avenir, 2009/10 n³0, p. 127-145. DOI : 10.3917/mav.030.0127

Distribution électronique Cairn.info pour Management Prospective Ed.

(C) Management Prospective Ed.. Tous droits réservés pour tous pays.

La reproduction ou représentation de cet article, notamment par photocopie, n'est autorisée que dans les limites des conditions générales d'utilisation du site ou, le cas échéant, des conditions générales de la licence souscrite par votre établissement. Toute autre reproduction ou représentation, en tout ou partie, sous quelque forme et de quelque manière que ce soit, est interdite sauf accord préalable et écrit de l'éditeur, en dehors des cas prévus par la législation en vigueur en France. II est précisé que son stockage dans une base de données est également interdit. 


\title{
Les défis de fins de carrière et la retraite: le cas du Québec
}

\author{
par Diane-Gabrielle Tremblay et Maryse Larivière
}

\section{Résumé}

Le vieillissement de la main-d'œuvre constitue un défi majeur. Le texte présente la situation démographique et du marché du travail, puis s'intéresse aux fins de carrière et aux aspirations des travailleurs vieillissants. Diverses formes d'aménagement du temps de travail, de retraite progressive et de télétravail pourraient éventuellement inciter des travailleurs à rester plus longtemps en emploi.

\section{Abstract}

Workforce aging is a major challenge. This text presents the demographic and labour market situation and then concentrates on the aspirations of workers and their last years of active life. Various forms of working time arrangements, of progressive retirement and telework could bring some workers to stay longer in employment.

Le vieillissement de la main-d'œuvre représente un enjeu majeur pour les acteurs du marché du travail et oblige la société québécoise à s'adapter à de nouvelles réalités, dont celle d'une main-d'œuvre plus âgée qu'auparavant et qui se renouvelle moins vite en raison d'une fécondité qui ne suffit pas à remplacer les générations et de l'allongement de l'espérance de vie.

Dans ce texte, nous tenterons de voir quelles mesures permettraient de prolonger la vie active des salariés, mais pour ce faire, il nous faut d'abord présenter quelques éléments sur la situation du marché du travail.

\section{La situation du marché du travail}

Selon l'Institut de la statistique du Québec (ISQ), il y aura de plus en plus de personnes âgées dans un Québec qui se dirige vers une diminution de sa population, laquelle sera réalité à compter de 2026 (Gauthier, 2007). En outre, l'allongement de la période de formation initiale et le retrait hâtif du marché du travail se sont traduits par la diminution du nombre d'années au travail. Cela se produit au moment même où la population d'âge actif (15 à 64 ans) s'accroît beaucoup plus lentement que pendant les dernières décennies (Gouvernement 


\section{REVUE \\ management \\ \& avenir}

du Québec, 2003). « Le vieillissement de la population concerne tout l'Occident, mais celui de la population du Québec se produit plus rapidement qu'ailleurs au Canada et aux États-Unis. L'écart entre le Canada et le Québec s'explique principalement par un solde migratoire québécois largement inférieur à celui du Canada. » (Gouvernement du Québec, 2003 : 6) Bien que depuis l'entrée en vigueur du nouveau congé parental québécois en 2006 (Tremblay, 2008), l'augmentation récente du taux de fécondité à 1,7 (par opposition à 1,45 auparavant) améliore les perspectives et retarde l'échéance, il reste que le vieillissement de la population québécoise se poursuivra, puisque le taux de remplacement des générations est de 2 .

Par ailleurs, la population des travailleurs âgés ne constitue pas un groupe homogène. La situation de ces personnes peut varier considérablement selon la région où elles vivent et le secteur d'activité dans lequel elles exercent leur métier ou leur profession. En fait, tout indique l'existence de difficultés particulièrement importantes de maintien en emploi ou de réintégration à l'emploi dans les régions-ressources et dans les secteurs d'activité en stagnation ou en déclin, qui ont du mal à évoluer dans un contexte où la concurrence internationale est forte. Selon les « indicateurs de mieux-être du Canada » (RHDSC, 2008), 57 \% des Canadiens de plus de 65 ans possèdent une source privée de revenu issue de placements et $62 \%$ bénéficient de régimes privés de pension et de REER (Régime enregistré d'épargne retraite), comparativement à 52 et $56 \%$ pour le Québec. Citant des données de 2001 et 2005, Lizée (2007) souligne les inégalités d'accès à ces systèmes : seul quatre travailleurs sur dix seraient couverts par un régime complémentaire de retraite et, en 2001 , une famille canadienne sur trois ne possédait aucun avoir de retraite.

De plus, la main-d'œuvre plus âgée est appelée à se féminiser et le vieillissement de la main-d'œuvre féminine présente des caractéristiques différentes de la maind'œuvre masculine. En plus d'une répartition des emplois fortement différenciée selon le sexe, les conditions de travail varient considérablement, au désavantage des femmes. En outre, leur participation croissante au marché du travail est combinée à un cycle de vie professionnel plus court mais, surtout, plus discontinu que celui des hommes. Cette situation a pour effet d'exposer davantage à la pauvreté les femmes de 55 ans et plus dans les cas où elles ne peuvent se maintenir en emploi ou réintégrer le marché du travail (Gouvernement du Québec, 2003). II faut toutefois reconnaître que les femmes des dernières décennies ont des parcours professionnels qui se rapprochent de plus en plus de ceux des hommes du point de vue de la participation au marché du travail, de sorte que c'est plutôt le fait qu'elles travaillent dans des secteurs moins rémunérateurs ou plus précaires qui les désavantage. Elles semblent avoir fait des progrès sur ce plan, mais des écarts subsistent dans certains groupes (Cloutier, 2008 et Cloutier et al., 2008). 


\section{Les défis de fins de carrière et la retraite : le cas du Québec}

Comme c'est le cas en Europe, le gouvernement du Québec prône une politique de vieillissement avec la Stratégie d'intervention à l'intention des travailleurs âgés de plus de 45 ans, visant le maintien ou le retour en emploi (Gouvernement du Québec, 2003). Les entreprises s'inquiètent de la pénurie de main-d'œuvre qualifiée bien que dans certains cas, les pénuries soient surtout appréhendées. Cela expliquerait l'importante croissance accordée à la question du maintien en activité de la main-d'œuvre vieillissante, bien que le Québec et le Canada présentent les taux d'activité parmi les plus élevés, dépassant en fait la moyenne de l'OCDE pour l'ensemble des 15-64 ans, et que certaines catégories de maind'œuvre ne voudront pas ou ne pourront pas rester en emploi au-delà de 50 ou 55 ans.

Figure : Taux d'activité des personnes de 15-64 ans selon le pays en 2007, incluant Canada et Québec.

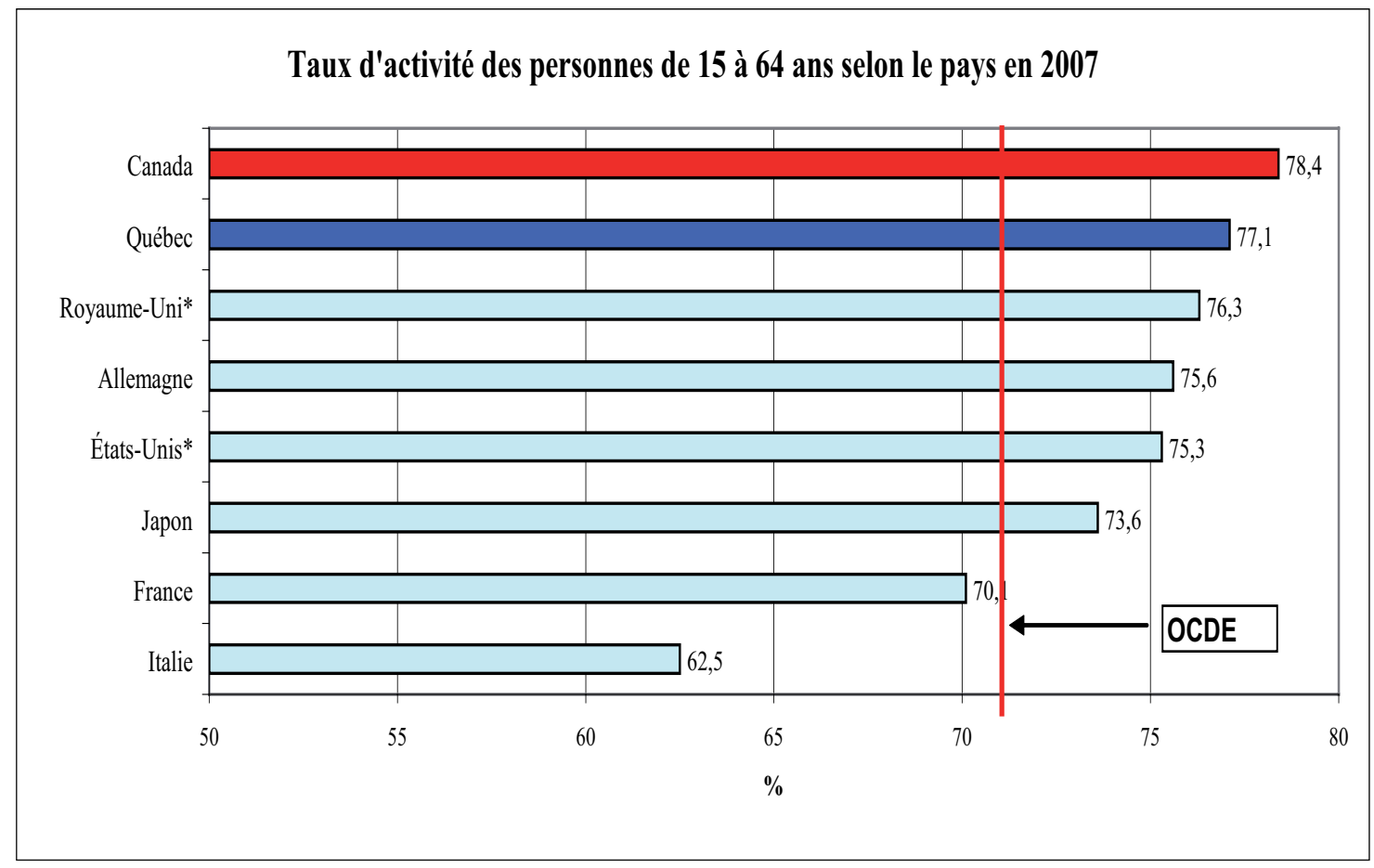

* : Pour le Royaume-Uni et les États-Unis, personnes de 16 à 64 ans.

Source: Présentation d'André Grenier à l'ASDEQ (Association des économistes du Québec), 5 février 2009. Sources premières : OCDE, OECD Employment Outlook 2008 et Statistique Canada, Enquête sur la population active.

Le Québec et le Canada présentent des taux d'activité des jeunes et des plus âgés plus élevés que nombre d'autres pays, ce qui n'empêche pas les gouvernements d'être préoccupés par le taux d'activité de fin de carrière et de souhaiter le voir augmenter davantage, essentiellement pour faire face aux coûts de la santé et de la sécurité sociale, ainsi qu'aux déficits des finances publiques, dans les prochaines décennies. Certains individus peuvent aussi souhaiter prolonger leur activité; les données canadiennes indiquent qu'entre 1991 et 2007, la proportion de personnes de 45-49 ans envisageant de se retirer du marché du travail avant 


\section{REVUE \\ management \\ \& avenir}

60 ans a augmenté de 4 points de pourcentage, alors que la part envisageant une retraite à 65 ans ou plus a augmenté d'environ 7 points de pourcentage (Schellenberg et Ostrovsky, $2008: 11$ ).

Au Québec, le groupe en âge de travailler (15-64 ans) se réduit au fil des ans, alors que la catégorie des 65 ans et plus augmente en pourcentage et que celle de 0-14 ans diminue considérablement, de sorte que certains prévoient des déficits de population active. La situation est un peu moins problématique au Québec et au Canada qu'ailleurs, et notamment en Europe puisque le taux d'emploi y est globalement plus élevé, mais on craint malgré tout des pénuries de maind'œuvre. Nombre de pays européens ont eu des régimes de préretraite qui ont incité les travailleurs à quitter hâtivement le marché du travail, ce qui est moins généralisé au Québec et au Canada (Tremblay, Najem, Paquet, 2007).

Le nombre moyen d'heures de travail par travailleur et par année est aussi un aspect important lorsqu'on traite de la fin de carrière. Outre que l'activité dépend toujours en partie de la santé de l'économie et des entreprises, et non seulement du bon vouloir des individus, la figure 2 nous permet de constater que l'on travaille effectivement un plus grand nombre d'heures aux États-Unis et au Japon (quoique la tendance soit à la baisse). Par contre, les travailleurs québécois font davantage d'heures que les Français et les Suédois notamment. Tout dépend donc de la base de comparaison, mais la tendance est toutefois clairement à la baisse dans tous les pays et certains craignent que le Québec ne vive une pénurie de main-d'œuvre plus importante qu'ailleurs, en raison des horaires réduits de ses travailleurs (mais plus élevés que la France).

Figure 2. Nombre moyen d'heures de travail par travailleur et par année, Québec, France et autres pays, $1950-2000$

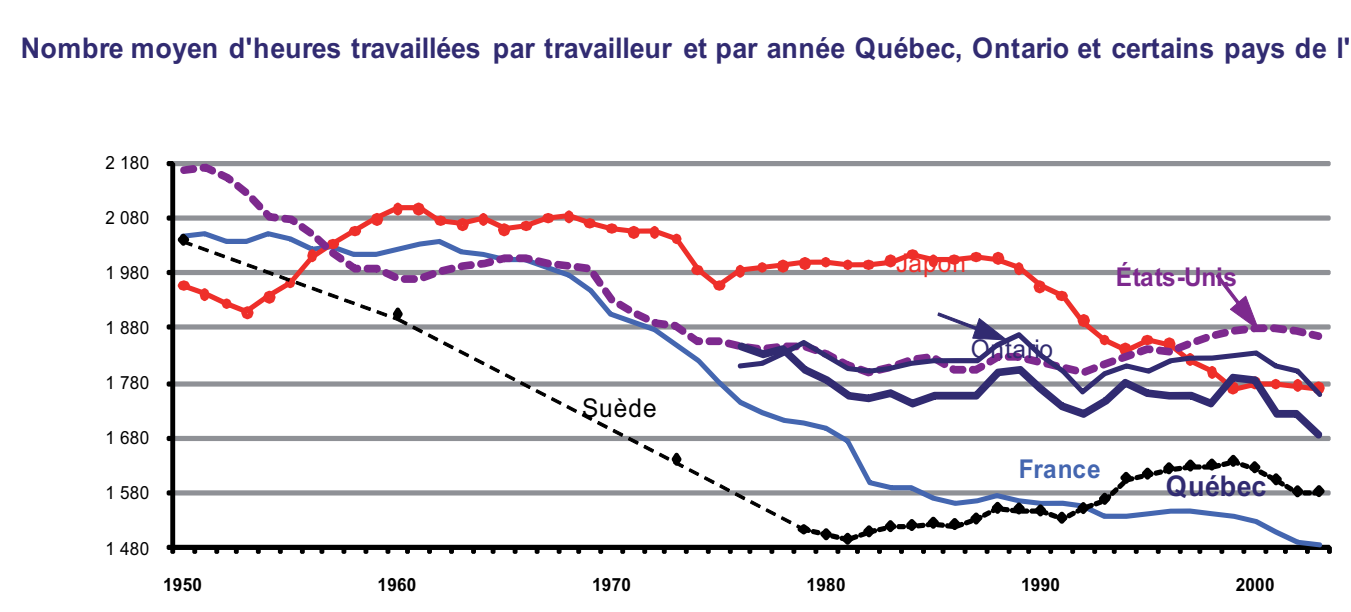

Source : B. Matte (2007). « Emploi et niveau de vie dans un contexte de vieillissement et de précarité des finances publiques », présentation à la conférence Halte à la retraite! De la culture de la retraite à la gestion des âges. reproduit dans Tremblay, Najem et Paquet, 2007. 


\section{Les défis de fins de carrière et la retraite : le cas du Québec}

Dans les pages qui suivent, nous étudierons les fins de carrière, dont différentes politiques de retraite progressive et de retour à l'emploi qui commencent à transformer le portrait des fins de carrière au Québec. II semble que des mesures d'aménagement du temps de travail ou de la fin de carrière, comme le télétravail et les horaires flexibles, pourraient permettre d'accroître le taux d'activité en offrant aux salariés des conditions qu'ils recherchent.

\section{La retraite progressive et le retour à l'emploi}

La retraite progressive constitue une avenue à explorer pour les employeurs confrontés à une pénurie de main-d'œuvre, mais aussi pour les salariés qui craignent une rupture trop soudaine avec le marché du travail. La retraite progressive consiste à se retirer graduellement du marché du travail en réduisant ou en aménageant ses heures de travail, en réduisant sa charge de travail ou, encore, en aménageant son poste de travail, sous l'angle ergonomique, afin de rendre le travail moins difficile.

Au Québec, il est maintenant possible pour les salariés de prendre une retraite progressive sans subir par la suite de pénalités dans leurs prestations de retraite. La stratégie gouvernementale vise à maintenir en emploi les travailleurs de 55 ans et plus et à encourager le travail des retraités qui le souhaitent et qui concluent une entente avec leur employeur à cet effet. « Le maintien au travail ou le retour au travail de la main-d'œuvre âgée dépend très étroitement de la flexibilité de l'organisation du travail et des programmes qui facilitent la transition vers la retraite. " (Asselin, 2007 : 218) En 2003, Emploi-Québec a publié une Stratégie d'intervention à l'intention des travailleuses et des travailleurs de 45 ans et plus en concertation avec le Comité aviseur pour les travailleuses et travailleuses de 45 ans et plus, la Commission des partenaires du marché du travail et le Conseil consultatif du travail et de la main-d'œuvre.

Par ailleurs, des améliorations sont apportées au Régime de rentes du Québec depuis 1998 pour favoriser la retraite progressive du marché du travail. " Malgré ces dispositions plus avantageuses, les conditions financières moins favorables des régimes complémentaires de retraite (régimes d'employeurs) peuvent inciter à prolonger la vie active. Parmi ces conditions, mentionnons la solvabilité précaire des régimes de retraite à prestations déterminées, la non-indexation des rentes de retraite ou l'indexation insuffisante. " (Asselin, $2007: 218$ ) Le gouvernement du Québec a modifié, tout récemment, les dispositions concernant la retraite progressive.

\subsection{Les mesures favorisant la retraite progressive}

La Régie des rentes du Québec a apporté deux modifications à son régime de retraite en 1997 afin de compenser la perte de salaire subie par une réduction 


\section{REVUE \\ management \\ \& avenir}

du temps de travail et pour ainsi faciliter la prise de retraite progressive. Les travailleurs indépendants sont cependant exclus de ces mesures. Ainsi, depuis le 1 er janvier 1998, un salarié ayant entre 55 et 70 ans peut réduire son temps de travail et continuer à cotiser au $R R Q$, comme si son salaire n'avait pas été réduit. Le salarié doit d'abord conclure une entente avec l'employeur et la Régie des rentes du Québec. II doit avoir un salaire annuel au moins égal à 3500 \$ après réduction de son temps de travail. Le salaire sur lequel le travailleur et l'employeur cotiseront ne doit pas dépasser deux fois et demie le salaire gagné après la réduction de son temps de travail (FADOQ, 2008). Du 1er janvier 1998 au 8 décembre 2006, seulement 40 adhésions ont été enregistrées (RRQ, 2007).

Un travailleur âgé entre 60 et 65 ans peut demander à son employeur de réduire d'au moins $20 \%$ son salaire après entente de retraite progressive et peut solliciter une rente de retraite anticipée du Régime de rentes pour compenser la perte de salaire. Les personnes de 65 ans et plus peuvent recevoir une rente de retraite de la $R R Q$ et continuer à travailler sans restriction. Selon les données de la Régie des rentes du Québec, du 1er janvier 1998 au 8 décembre 2006, 31310 personnes se sont prévalues de cette disposition (FADOQ, 2008).

\subsection{Les mesures concernant les régimes complémentaires de retraite}

Depuis 1997, à partir de 55 ans et avec l'accord de son employeur, un travailleur québécois peut, tout en continuant à travailler à temps partiel, réduire ses heures de travail pendant quelques années et toucher des prestations d'un régime privé de retraite. Le travailleur doit être à moins de 10 ans de l'âge de la retraite normale prévu par son régime privé, généralement 65 ans, et avoir 69 ans ou moins. A contrario des mesures précédentes, celle-ci n'a pas à être approuvée par la Régie des rentes du Québec. Le participant peut continuer à accumuler du service en travaillant à temps réduit lors de sa retraite progressive. II s'agit d'une prestation anticipée dont le montant pour une année ne doit pas dépasser le plus petit des montants suivants :

- « $70 \%$ de la perte de revenu provenant de la réduction des heures de travail

- $40 \%$ du maximum des gains admissibles (MGA) 17960 \$ pour 2008

- la valeur de la rente acquise dans le Régime complémentaire de retraite $(R C R) »(F A D O Q, 2008)$.

La valeur des montants forfaitaires versés au participant pendant la période de retraite progressive réduira la valeur de sa rente lorsque ce dernier prendra sa retraite complète (FADOQ, 2008). 


\section{Les défis de fins de carrière et la retraite : le cas du Québec}

\subsection{Les modifications relatives à la retraite progressive}

Le gouvernement du Québec a déposé en avril 2008 le projet de loi 68 en vue de modifier la Loi sur les régimes complémentaires de retraite (Loi RCR) pour profiter des modifications apportées par le fédéral au Règlement de l'impôt sur le revenu relativement à la retraite progressive. Les mesures concernant le supplément de rente s'appliquent dès 2009, en utilisant les gains admissibles de 2008, aux régimes de retraite du secteur privé, des sociétés d'État, des municipalités et des universités. Le projet de loi contient des mesures qui permettent le versement d'une rente pendant une période d'emploi pour un participant à un régime à prestations déterminées, et le versement d'une prestation dans le cas d'un régime à cotisations déterminées. Avant cette modification, un travailleur qui recevait une rente de retraite du $R R Q$, tout en y cotisant, ne pouvait accumuler de droits additionnels au titre du RRQ que dans des cas limités. Le projet de loi modifie cette situation en accordant aux travailleurs le droit à un supplément de rente et les gains admissibles d'une année donneront droit à un supplément de rente à compter du 1er janvier de l'année suivante. Si le travailleur demeure au travail et cotise au $R R Q$ plusieurs années après sa retraite, il pourra avoir droit à plusieurs suppléments de rente. Le supplément de rente sera traité comme une rente de retraite, il sera donc versé durant la vie du travailleur et sera indexé chaque année (FADOQ, 2008).

\section{Les régimes à prestations déterminées}

Le participant à un régime à prestations déterminées (PD) ou au volet PD d'un régime à double volet qui demeure à l'emploi pourra recevoir une prestation s'il satisfait aux conditions suivantes:

- avoir au moins 60 ans ou

- avoir au moins 55 ans et avoir droit à une rente non réduite et

- ne pas avoir atteint l'âge de 65 ans.

Une entente avec l'employeur est requise, mais le participant n'aura pas obligatoirement à diminuer son temps de travail ni à subir une diminution de salaire. Toutefois, la prestation de retraite progressive ne devra pas excéder $60 \%$ du montant annuel de la rente à laquelle le participant aurait eu droit s'il avait pris sa retraite à la date de la demande, ou de la rente à laquelle il a droit s'il reçoit déjà une rente. Certaines prestations seront exclues de ce calcul comme les rentes constituées avec les cotisations excédentaires ou les cotisations volontaires, mais les prestations de raccordement seront incluses.

La prestation prend fin lorsque le participant atteint 65 ans. Un participant ne peut recevoir en même temps une prestation de retraite progressive et une autre prestation au titre du régime, sauf dans le cas d'une prestation de retraite progressive payable dans le cadre d'un volet " cotisations déterminées " (CD) du régime. Si le participant reçoit déjà une rente de retraite du régime au moment 


\section{REVUE \\ management \\ \& avenir}

où il demande la prestation de retraite progressive, le versement de la rente sera suspendue. Selon la position de la Régie des rentes, le régime peut permettre l'accumulation de droits à retraite pendant la période de retraite progressive.

Cependant, la rémunération reçue par le participant pendant la période de retraite progressive ne pourra être prise en compte aux fins du calcul de la rente de retraite « finale » du participant, sauf si cela avantage le participant.

Pour les participants qui recevaient déjà une rente avant la période de retraite progressive :

- la suspension de la rente de retraite se terminera à la fin de la période de retraite progressive;

- si le participant recevait une rente anticipée réduite, la réduction sera calculée à nouveau à la fin de la période de retraite progressive;

- si des cotisations sont versées au régime pendant la période de retraite progressive, la rente de retraite sera augmentée d'une rente dont la valeur correspondra au moins aux cotisations salariales avec intérêts (FADOQ, 2008).

\section{Les régimes à cotisations déterminées}

Les régimes de retraite qui contiennent une composante à cotisations déterminées pourront offrir le versement d'une prestation pendant la période de retraite progressive mais ne seront pas tenus de le faire. Pour bénéficier d'une telle prestation, le participant devra avoir entre 60 et 65 ans et conclure une entente à cet effet avec son employeur. Les modalités de calcul et de paiement de la prestation seront prévues par le régime. Toutefois, le montant annuel de la prestation ne pourra excéder $60 \%$ du plafond de revenu viager que le participant pourrait recevoir d'un fonds de revenu viager ${ }^{8}$. Les prestations versées à un participant pendant la période de retraite progressive viendront réduire le solde de son compte à cotisations déterminées.

La retraite progressive n'est pas un droit automatique pour l'employé. L'employeur devra modifier le régime pour la prévoir et conclure une entente avec l'employé pour la mettre en œuvre. "Si le texte du régime prévoit des modalités précises relatives à la retraite progressive - même si le droit de bénéficier de la retraite progressive est lié au consentement de l'employeur - alors le texte du régime n'aura pas à être modifié chaque fois qu'un employé se prévaut de ces modalités. Par contre, dans la mesure où le texte du régime laisse les modalités à la discrétion de l'employeur, le texte du régime devra être modifié chaque fois qu'un employé se prévaudra de la retraite progressive » (Mercer, 2008).

8. Le fonds de revenu viager (FRV) est un fonds enregistré de revenu de retraite (FERR) particulier dans lequel une personne peut transférer les sommes provenant de son régime complémentaire de retraite ou de son compte de retraite immobilisé (CRI). À la différence d'un FERR où il n'existe aucun plafond, il n'est pas possible de retirer d'un FRV plus que le maximum autorisé chaque année. Depuis le 1er janvier 1998, il n'est plus obligatoire d'acheter une rente viagère avec le FRV à l'âge de 80 ans. (Régime des rentes du Québec. http://www.rrq.gouv.qc.ca/fr/programmes/rcr/CRI_FRV/FRV/ Page consultée le 4 décembre 2008.) 


\section{Les défis de fins de carrière et la retraite: le cas du Québec}

Les mesures offrent une certaine flexibilité à l'employeur qui pourra définir les conditions conformes à ses objectifs compte tenu de ses besoins de maind'œuvre et de l'ensemble de ses coûts de même que les coûts du régime de retraite. Par contre, la prestation de retraite progressive dans les régimes à cotisations déterminées sera financée à même les droits déjà accumulés par les employés et elle ne devrait donc pas représenter un coût supplémentaire pour les employeurs (FADOQ, 2008).

\subsection{Les mesures dans les conventions collectives}

Le passage d'un emploi à plein temps à la pleine retraite représente une étape importante de la vie d'un travailleur, mais les aspirations en matière d'aménagement et de réduction du temps de travail (ARTT) en fin de carrière le sont tout autant (Tremblay, 2007; Tremblay, 2008a). Par ailleurs, dans beaucoup de milieux de travail, il semble difficile d'avoir accès à des mesures permettant une plus grande flexibilité dans l'organisation du travail (Tremblay, 2008b; Tremblay et al., 2008). Comme nous le verrons dans cette section, ce sont surtout les travailleurs avec de bons emplois qui peuvent profiter de mesures d'aménagement ou de réduction du temps de travail dans le cadre d'une retraite progressive.

Nous avons mentionné précédemment qu'il existe un programme public destiné à favoriser le recours à des mesures d'aménagement et de réduction du temps de travail (ARTT). Cependant, il semble que les entreprises qui mettent en place de telles mesures le font parfois sans l'aide de l'État. Selon le Réseau FADOQ, le recours au programme d'ARTT d'Emploi-Québec reste encore très faible, puisque 30 entreprises seulement y ont fait appel entre avril et décembre 2002. S'inspirant notamment des travaux de Bellemare et al. (1998) et de Tremblay (2003), le Réseau FADOQ (2008) explicite ce constat.

«Parmi les faiblesses relevées à propos du programme d'ARTT, des chercheurs identifient l'insuffisance des ressources allouées (humaines, financières, techniques), l'absence d'une campagne promotionnelle d'envergure permettant de faire connaître le programme, le caractère trop défensif des interventions, le recours trop exclusif aux mesures de préretraite et certains problèmes reliés à l'assurance-emploi et à la Régie des rentes du Québec »(FADOQ, 2008).

Suivant une étude de Fourzly et Gervais (2002), de nombreuses conventions collectives, au Canada, contiennent des clauses concernant les travailleurs âgés qui approchent de la retraite. En général, le but principal est d'inciter les travailleurs à prendre une retraite anticipée en leur offrant divers stimulants financiers. Toutefois, dans le contexte actuel caractérisé par une pénurie de travailleurs qualifiés et un vieillissement rapide de la main-d'œuvre, plusieurs conventions prévoient des moyens de maintenir les travailleurs âgés sur le marché du travail ou de faciliter leur transition vers la retraite. Plusieurs conventions collectives 


\section{REVUE \\ management \\ \& avenir}

comportent des mesures spécifiquement conçues pour faciliter la transition à la retraite, alors que d'autres mesures sont prises à l'amiable ou ne relèvent pas de la négociation collective, mais simplement de politiques de gestion (Fourzly et Gervais, 2002). Nous avons analysé les différentes clauses recensées par les auteurs en nous intéressant plus particulièrement au Québec.

\section{Les congés de préretraite}

Les congés de préretraite peuvent aider les employés âgés à se préparer à la retraire et à faciliter la transition travail-retraite en leur permettant de " s'exercer à la vie de retraité » avant de quitter leur emploi (Malcolm, 1985). Suivant Fourzly et Gervais, en janvier 1998, environ 6,5\% des principales conventions collectives canadiennes, touchant $9,2 \%$ des employés (206 797 employés), prévoyaient des congés de préretraite. II semble que les congés qu'offrent ces clauses sont très variables, allant des congés annuels de la dernière année civile de service, jusqu'à une année de congé payée. D'autres clauses prévoient des mesures offrant des avantages tels que les congés annuels, des crédits de congés de maladie, des indemnités de maladie ainsi que des allocations du régime de retraite. La durée et les conditions des congés de préretraite varient selon les conventions et l'admissibilité dépend habituellement de l'âge de l'employé, de ses années de service ou d'une combinaison des deux. Par ailleurs, les employés qui quittent leur emploi avant la fin de l'année d'acquisition des congés n'ont droit qu'à une proportion de la paye de vacances, sauf si la convention stipule clairement le contraire. Il en est de même lorsqu'un employé qui part à la retraite doit recevoir l'intégralité de sa paye de vacances annuelles (Fourzly et Gervais, 2002).

Nous présentons ci-dessous un exemple de clause sur le congé de préretraite.

\section{Les programmes de préparation à la retraite}

Fourzly et Gervais (2008) ont également trouvé des clauses relatives à des services de conseil dans $13,4 \%$ des principales conventions collectives canadiennes visant $19,2 \%$ des employés (431 601 employés). II semble que ces services n'offrent pas tous une aide spécifiquement liée à la retraite, bien qu'il existe néanmoins certains programmes qui offrent de l'aide aux travailleurs sur le point de prendre leur retraite. Ces programmes touchent principalement des domaines tels que "les soins de santé, la planification financière, le logement, l'adaptation au nouveau mode de vie, les questions juridiques, l'organisation du temps de loisirs et les techniques de recherche d'emploi en vue d'une seconde carrière » (Fourzly et Gervais, 2002 : 211). Dans presque tous les cas recensés par les auteurs, les clauses offrant un programme ou un séminaire de conseil avant la retraite sont relativement brèves et présentent peu de détails, mais elles stipulent que le service sera financé et, si possible, fourni par l'employeur. Ces programmes peuvent aider les travailleurs âgés à se préparer à relever de 


\section{Les défis de fins de carrière et la retraite : le cas du Québec}

nouveaux défis tout en atténuant leurs craintes éventuelles (Fourzly et Gervais, 2002).

\section{La retraite échelonnée}

Pour Fourzly et Gervais (2002), la retraite échelonnée comprend tout programme permettant de réduire progressivement le temps et la charge de travail au lieu de passer brutalement d'un emploi à plein temps à la retraite. Les auteurs ont trouvé deux formules de retraite échelonnée (ou de "retraite graduelle ») dans les conventions collectives : une réduction graduelle des heures (ou des jours) de travail avant la retraite, ou un emploi à temps partiel après le départ à la retraite pour les pensionnés qui souhaitent continuer à travailler. Les programmes de retraite échelonnée peuvent permettre à l'employeur de conserver des travailleurs âgés qualifiés qui normalement quitteraient leur emploi dans des secteurs particuliers où il y a pénurie de main-d'œuvre qualifiée, en plus de lui permettre de réduire les coûts de main-d'œuvre. La retraite échelonnée présente aussi des avantages pour les employés âgés; elle leur permet de s'adapter progressivement à la retraite tout en conservant un revenu plus élevé que s'ils devaient compter uniquement sur leur pension.

\section{La retraite graduelle}

Au fil des ans, les programmes de préretraite ou de retraite graduelle s'inscrivent de façon plus importante dans les conventions collectives. Ces programmes permettent aux travailleurs âgés de réduire progressivement leur charge de travail, de façon générale, en réduisant leurs heures de travail sur une certaine période avant de partir à la retraite et de toucher leur pension. La période précédant la retraite au cours de laquelle un employé peut réduire sa charge de travail est également variable, pouvant aller de un à cinq ans. Pour les auteurs, les programmes visent un double but: premièrement, faciliter la transition du travail à plein temps à la retraite et, deuxièmement, offrir aux jeunes travailleurs la possibilité d'accéder à un emploi à plein temps.

La convention collective de l'Université Concordia contient également une clause relative à un « arrangement particulier de réduction du temps de travail avant la retraite ». (Fourzly et Gervais, $2002: 214$ ) Bien que cet arrangement ne semble pas aussi avantageux financièrement que le programme de retraite graduelle décrit précédemment, il offre une autre forme de flexibilité aux travailleurs âgés qui approchent de la retraite.

\section{Les possibilités de travail après l'âge de la retraite}

Quelques organismes ont mis en place des programmes offrant aux travailleurs âgés, en particulier à ceux dont les compétences et l'expérience sont très recherchées, la possibilité de continuer à travailler au-delà de l'âge normal de la 


\section{REVUE \\ management \\ \& avenir}

retraite. Ces programmes permettent de différer la date de la retraite ou d'avoir un travail à temps partiel ou temporaire après avoir pris sa retraite. Quoiqu'on trouve surtout ces programmes dans le secteur des services, d'où les syndicats sont la plupart du temps absents, certaines conventions collectives, très peu nombreuses, comportent des dispositions concernant le travail après la retraite.

\section{La retraite différée}

Sous réserve de l'approbation de l'employeur ou d'une preuve d'aptitude au travail, plusieurs conventions autorisent les employés à prolonger leur vie active au-delà de l'âge normal de la retraite. Bien qu'aboli au Québec, l'âge de la retraite obligatoire était, jusqu'à tout récemment, fixé à 65 ans dans la majorité des provinces et des territoires du Canada. Les conditions d'emploi demeurent essentiellement les mêmes que celles des employés permanents.

\section{Le travail après la retraite}

Fourzly et Gervais (2002) ont recensé d'autres conventions permettant à certains employés de conserver leur emploi, en général à temps partiel, après la date de la retraite. Cette disposition permet aux travailleurs âgés de bénéficier d'un ajout de revenu à leur pension de retraite et de conserver un certain nombre d'avantages sociaux.

II semble que les principales conventions collectives canadiennes reflètent la nécessité de préparer la fin de la vie professionnelle des travailleurs âgés et comportent des dispositions qui traitent précisément de la transition travail-retraite (Fourzly et Gervais (2002). Parmi les mesures d'aménagement des conditions de travail, il en est une qui est demandée de manière presque unanime par les travailleurs, c'est celle de la réduction du temps de travail au cours des années de fin de carrière. II s'agit principalement d'envisager un développement du travail à temps partiel, ou une prise de retraite progressive ou un retrait progressif d'activité. Le principal but visé par les travailleurs est de pouvoir regagner une certaine maîtrise de l'utilisation de leur temps, de choisir leur horaire, d'introduire de la souplesse dans leur horaire, de pouvoir réduire progressivement leur rythme et leur temps de travail avant de quitter définitivement leur emploi (Tremblay et Genin, 2009 ; FADOQ, 2007 ).

Comme le montrent Fourzly et Gervais (2002), les clauses contractuelles relatives aux congés ou aux vacances de préretraite et les programmes de counselling en vue de la retraite démontrent la volonté des entreprises d'en tenir compte. Les conventions collectives prévoient de plus en plus souvent des programmes qui permettent aux employés de passer à la retraite en réduisant graduellement leur nombre d'heures de travail. Or si les mesures de réduction du temps de travail sont en partie appliquées dans les très grandes entreprises, comme l'ont montré Fourzly et Gervais, elles ne sont pratiquement pas offertes dans les PME, où l'on retrouve pourtant $70 \%$ des emplois. Aucun aménagement du temps de 


\section{Les défis de fins de carrière et la retraite : le cas du Québec}

travail ne tient compte du vieillissement dans les PME, si ce n'est de très rares cas de retraites progressives où le travail est étalé sur quatre jours avec une cinquième journée compensée par le Régime des rentes du Québec (Bellemare et al., 1998 ; FADOQ, 2007).

Même si certains employés préfèrent continuer à travailler après l'âge normal de la retraite, ils peuvent alors bénéficier de dispositions qui leur permettent de reporter la date de la retraite et de profiter de possibilités de travail à temps partiel après la retraite. Toutefois, la notion même de vieillissement en emploi est relative au type de production, au type de compétences exigées, à l'organisation du travail, alors que les travailleurs tentent de concilier maintien de la productivité, qualité du travail et préservation de la santé (Lagacé, 2007). Selon nombre d'études, on vieillit différemment selon l'emploi occupé (Bellemare et al., 1998). Mais encore, le fait de choisir l'une de ces formules peut avoir des répercussions sur les avantages sociaux de l'employé pendant qu'il conserve son emploi, tant sur le plan des cotisations et de l'accès aux régimes qu'en ce qui concerne le salaire cotisable qui servira de base au revenu qu'il touchera pendant sa retraite (Fourzly et Gervais, 2002). Se pose alors la question de l'articulation entre les mesures de réduction du temps de travail et l'accès aux programmes de compensation financière qui vont combler tout ou une partie du manque à gagner, qu'il s'agisse de l'assurance-emploi, de l'aide sociale (sécurité du revenu) ou des régimes de retraite ou de préretraite (Tremblay et Genin, 2009b ; FADOQ, 2008, 2007).

\section{De bonnes pratiques pour les fins de carrière ?}

Avec la crise financière, mais aussi compte tenu de l'amélioration de la santé chez les 45 ans et plus et de l'espérance de vie augmentée (Asselin, 2007; FADOQ, 2008), certains retraités pourraient retourner en emploi après une période de retraite, soit parce qu'ils manquent de revenus, soit parce qu'ils souhaitent retrouver une vie sociale dans un milieu de travail (Fusulier et al., 2008). On peut susciter l'intérêt d'un plus grand nombre de travailleurs âgés quant à leur maintien en emploi ou à leur retour sur le marché de l'emploi en bonifiant l'aménagement du temps de travail et les tâches (Tremblay et Genin, 2009b ; FADOQ, 2008).

Dans un mémoire du Réseau FADOQ, on propose diverses mesures afin de modifier l'organisation du travail de manière à permettre aux travailleurs d'expérience de rester plus longtemps en emploi et de transmettre ainsi leur savoirfaire à la relève (FADOQ, 2007). Des améliorations pourraient être apportées en misant sur la transformation des emplois. Par exemple, on pourrait offrir des conditions de travail mieux adaptées comme des postes moins durs, des mesures ergonomiques, l'assignation d'un rôle de tuteur, en plus des aménagements du temps de travail tels le temps partiel, l'horaire flexible, le partage du travail et le désengagement progressif. 


\section{REVUE \\ management \\ \& avenir}

D'autres améliorations possibles concernent la gestion des carrières comme l'accès aux évaluations de performance, la mobilité verticale et latérale, l'élargissement des tâches et le développement de la polyvalence, ainsi que la gestion à long terme des emplois et la planification des carrières. De plus, la formation des travailleurs âgés est un aspect important susceptible de prolonger leur vie active. Le Réseau FADOQ propose une approche individualisée qui reconnaît les acquis non sanctionnés par un diplôme, les pratiques de formation adaptées et la formation tout au long de la carrière (FADOQ, 2007). En ce qui à trait aux mesures de formation ou de mentorat, il y a la Loi favorisant le développement de la formation de la main-d'œuvre (dite loi du $1 \%$ ) qui oblige les employeurs dont la masse salariale annuelle dépasse 1 million de dollars à investir l'équivalent d'au moins $1 \%$ de cette masse salariale pendant l'année dans la formation de leur personnel. Sinon, ils doivent verser le montant non investi au Fonds national de la formation de la main-d'œuvre afin d'améliorer certaines activités liées à la formation. Voilà une mesure concrète qu'une entreprise pourrait proposer à ses travailleurs vieillissants pour le prolongement de leur vie active.

Avant de conclure, nous allons présenter quelques données issues d'une enquête que nous avons menée récemment et qui permet de voir les préférences des salariés québécois en matière de fins de carrière. On constate que les mesures qui intéressent le plus de gens (total de très intéressant et intéressant) sont la retraite progressive, les heures flexibles en fin de carrière, les vacances annuelles plus longues, les horaires flexibles, la retraite et le maintien en emploi à temps partiel, des congés supplémentaires, le télétravail à temps partiel, etc. Ce qu'il faut retenir, c'est qu'il existe un grand nombre de mesures qui permettraient de retenir en emploi les travailleurs vieillissants, de sorte que les entreprises et les États qui veulent agir sur ce plan peuvent le faire. 


\section{Les défis de fins de carrière et la retraite : le cas du Québec}

Tableau 1 : Mesures ou incitatifs pour rester plus longtemps en emploi Question: Si les measures suivantes vous étaient offertes, lesquelles vous inciteraient (ou vous ont incité) à rester plus longtemps en emploi ?

\begin{tabular}{|c|c|c|c|c|c|}
\cline { 2 - 5 } \multicolumn{1}{c|}{} & $\begin{array}{c}\text { Très } \\
\text { intéressant }\end{array}$ & Intéressant & Neutre & $\begin{array}{c}\text { Pas très } \\
\text { intéressant }\end{array}$ & $\begin{array}{c}\text { Pas du tout } \\
\text { intéressant }\end{array}$ \\
\hline Retraite progressive & $50,49 \%$ & $25,73 \%$ & $11,65 \%$ & $4,37 \%$ & $7,77 \%$ \\
\hline Heures flexibles en fin de carrière & $46,58 \%$ & $30,59 \%$ & $10,50 \%$ & $3,65 \%$ & $8,68 \%$ \\
\hline Vacances annuelles plus longues & $42,58 \%$ & $28,23 \%$ & $12,44 \%$ & $5,74 \%$ & $11,00 \%$ \\
\hline $\begin{array}{c}\text { Horaires flexible (avec plage fixe dans } \\
\text { la journée) }\end{array}$ & $43,96 \%$ & $22,71 \%$ & $13,53 \%$ & $7,25 \%$ & $12,56 \%$ \\
\hline $\begin{array}{c}\text { Retraite et temps partiel dans la meme } \\
\text { organisation }\end{array}$ & $37,62 \%$ & $33,33 \%$ & $12,38 \%$ & $5,24 \%$ & $11,43 \%$ \\
\hline $\begin{array}{c}\text { Congés supplémentaires } \\
\text { Possibilité de télétravailler quelques } \\
\text { jours par semaine }\end{array}$ & $30,50 \%$ & $32,50 \%$ & $17,50 \%$ & $6,50 \%$ & $13,00 \%$ \\
\hline Travail à temps réduit volontaire & $28,14 \%$ & $26,13 \%$ & $24,12 \%$ & $7,04 \%$ & $14,57 \%$ \\
\hline $\begin{array}{c}\text { Semaine réduite à 4 jours (payé 4 } \\
\text { jours) }\end{array}$ & $24,64 \%$ & $25,12 \%$ & $22,22 \%$ & $11,11 \%$ & $16,91 \%$ \\
\hline $\begin{array}{c}\text { Retraite et activité dans une autre } \\
\text { organisation }\end{array}$ & $22,92 \%$ & $22,40 \%$ & $27,60 \%$ & $9,38 \%$ & $17,71 \%$ \\
\hline \begin{tabular}{c} 
Journées de travail à temps réduit \\
\hline $\begin{array}{c}\text { Semaine comprimée à 4 jours } \\
\text { (remuneration normale) }\end{array}$
\end{tabular} & $22,17 \%$ & $25,12 \%$ & $22,17 \%$ & $8,37 \%$ & $22,17 \%$ \\
\hline $\begin{array}{c}\text { Travail partagé volontaire (deux } \\
\text { employés travaillant à temps partriel) }\end{array}$ & $20,60 \%$ & $28,14 \%$ & $18,59 \%$ & $9,55 \%$ & $23,12 \%$ \\
\hline $\begin{array}{c}\text { Possibilité de télétravailler à plein } \\
\text { temps }\end{array}$ & $14,29 \%$ & $13,78 \%$ & $23,47 \%$ & $14,29 \%$ & $34,18 \%$ \\
\hline
\end{tabular}

Source: Tremblay et Genin, 2009a

Nous allons présenter rapidement quelques autres éléments qui ressortent de cette enquête et qui donnent des indications sur les préférences pour la fin de carrière (Tremblay et Genin, 2009a) :

- Les répondants sont globalement opposés à l'idée de faire passer l'âge légal de la retraite à 67 ans, mais ils pensent dans une large majorité que l'on peut fournir un travail de qualité après 65 ans (âge normal, mais non obligatoire de la retraite au Canada).

- Les répondants sont beaucoup plus ouverts à des dispositifs permettant un passage progressif à la retraite plutôt qu'un recul de l'âge légal de la retraite.

- On note une forte demande pour des aménagements du temps de travail en fin de carrière : retraite progressive, temps partiel, possibilité de travailler à domicile... 


\section{REVUE \\ management \\ \& avenir}

- Une large majorité des répondants ont un revenu inférieur à la retraite par rapport à ce qu'ils avaient avant. Cependant, plus de $70 \%$ se déclarent satisfaits de leur revenu (notons que ces réponses ont été enregistrées avant le déclenchement de la crise financière).

- Près d'un tiers des répondants retraités sont retournés sur le marché du travail depuis leur retraite. Notons que ces réponses ont été enregistrées avant le déclenchement de la crise financière, et qu'il est possible que ce taux augmente dans les mois à venir.

- L'ensemble des répondants ont globalement une vision positive de la retraite.

- Les répondants encore en emploi se déclarent à $80 \%$ satisfaits de leur emploi; pourtant près des deux tiers disent avoir hâte ou très hâte d'être à la retraite.

\section{Conclusion}

Au cours des dernières décennies, la participation des travailleurs plus âgés au marché du travail s'est transformée et, désormais, la retraite n'est pas nécessairement un événement ponctuel, mais bel et bien une succession d'événements d'entrée et de sortie possibles avant la retraite définitive. Un premier aperçu de la situation de ce groupe de travailleurs sur le marché de l'emploi au Québec a été rendu possible grâce à des indicateurs comme le taux d'emploi, le taux d'activité et le taux de chômage pour une population donnée. Nous avons constaté que la population des travailleurs âgés ne constitue pas un groupe homogène et c'est un aspect qu'il faut retenir dans tous les pays où l'on se préoccupe du vieillissement de la main-d'œuvre. En effet, on a souvent tendance à parler de la main-d'œuvre âgée comme d'un groupe homogène et les données présentées montrent bien que ce n'est pas le cas; du coup, les aspirations et décisions relatives à la fin de la vie active peuvent varier considérablement selon le secteur d'activité, le taux de scolarité, la catégorie socio-professionnelle, la taille de l'entreprise où elles exercent leur métier ou leur profession et, par le fait même, selon la situation financière de celle-ci. Par ailleurs, tout indique l'existence de difficultés particulièrement importantes de maintien en emploi ou de réintégration en emploi dans les régions-ressources et dans les secteurs d'activité en stagnation ou en déclin, qui ont du mal à évoluer dans un contexte de forte concurrence internationale. Ici encore, c'est une réalité qui ne touche pas que le Québec ; dans pratiquement tous les pays, et peut-être même encore davantage en Europe et en France, les travailleurs âgés ont de la difficulté à rester ou à se réintégrer sur le marché du travail.

Les données issues de l'enquête que nous avons menée permettent de voir les préférences des salariés québécois en matière de fins de carrière et l'on peut imaginer que la diversité de l'offre de mesures pourrait permettre de prolonger la carrière de nombreux travailleurs, au Québec comme ailleurs. Nous avons 


\section{Les défis de fins de carrière et la retraite: le cas du Québec}

vu que les mesures qui intéressent le plus de gens sont la retraite progressive, les heures flexibles en fin de carrière, les vacances annuelles plus longues, les horaires flexibles, la retraite et le maintien en emploi à temps partiel, des congés supplémentaires, le télétravail à temps partiel, etc. Il existe clairement un grand nombre de mesures qui permettraient de retenir en emploi les travailleurs vieillissants, de sorte que les pays qui veulent agir sur ce plan peuvent certes le faire.

II faut noter que cette recherche présente certaines limites, puisqu'elle a été menée en collaboration avec un organisme représentant les travailleurs de 50 ans et plus, mais n'est pas parfaitement représentative de la population. II n'existe malheureusement pas de données représentatives de la population québécoise ou canadienne, mais il serait certes intéressant de poursuivre les recherches pour tenter d'en obtenir, en nous assurant éventuellement la collaboration de I'Institut de la statistique du Québec ou un autre organisme afin de réaliser une enquête de plus grande envergure.

Dans un contexte de vieillissement des travailleurs et de pénuries de maind'œuvre appréhendées dans plusieurs secteurs, l'aménagement et la réduction du temps de travail en fin de carrière apparaissent, notamment, comme des options permettant de prolonger la vie active de certains salariés et, de fait, nos travaux récents montrent que c'est effectivement l'ARTT qui peut le plus inciter les travailleurs à rester en emploi (Tremblay et Genin, 2009 a et b ; Tremblay, Najem, 2008). Il va sans dire que ce type de mesure n'intéressera pas les salariés de tous les secteurs, plus particulièrement ceux dont la charge de travail s'est alourdie au cours des dernières années ou dont les conditions de travail ont été suffisamment difficiles pour qu'il soit peu probable qu'ils envisagent de prolonger leur vie active (Bellemare et al., 1998; Tremblay, 2007). Cependant, on observe déjà que certains retraités retournent en emploi après une période de retraite, soit parce qu'ils manquent de revenus, soit parce qu'ils souhaitent retrouver une vie sociale dans un milieu de travail. En améliorant de façon notable l'aménagement du temps et des tâches, en offrant le télétravail ou la préretraite, il semble possible de faciliter la prolongation de l'activité sur le marché du travail et, ainsi, de faire face au défi du vieillissement de la population et de la main-d'œuvre, tout comme aux difficultés financières de certains. II faut toutefois approfondir la recherche sur les fins de carrière, les motivations des individus et les formes d'activité souhaitées, ce que nous continuerons de faire dans nos recherches au cours des prochaines années ${ }^{9}$. II serait particulièrement intéressant d'étudier les différences entre les femmes et les hommes, les secteurs masculins et féminins, et de voir aussi comment les situations familiales peuvent influer sur les choix en fin de carrière.

9. Notamment dans le cadre du projet ARUC (Alliance de recherche université-communauté, programme de recherche financé par le Conseil de recherches en sciences humaines du Canada) sur la gestion des âges et des temps sociaux. 


\section{REVUE management \& avenir}

\section{Bibliographie}

Asselin, S. (2007). "En fin de vie active, conserver un lien avec le travail », dans Hervé Gauthier (dir.). Vie des générations et personnes âgées : aujourd'hui et demain. Québec, Institut de la statistique du Québec. Pp. 217-252.

Bellemare, D., Poulin-Simon L. et D.-G. Tremblay. (1998). Le paradoxe de l'âgisme dans une société vieillissante. Québec, Presses de l'université du Québec. 265 p.

Cloutier, Luc. (2008). La qualité de l'emploi au Québec, développements conceptuels et création d'une typologie. État actuel de la réflexion. Québec, Institut de la statistique du Québec, 47 p. http://www.stat.gouv.qc.ca/publications/remuneration/qualite_emploi.htm. Page consultée le 26 septembre 2008.

Cloutier, Luc, Paul Bernard et Diane-Gabrielle Tremblay. (2009). «Qualité de l'emploi et inégalités entre les genres : des changements marquants au Québec au cours de la dernière décennie », A paraître dans Studies in Social Justice, décembre 2009.

Cloutier, Luc, Paul Bernard et Diane-Gabrielle Tremblay. (2008). La qualité de l'emploi des femmes et des hommes au Québec; une étude de son évolution à l'aide d'une nouvelle typologie. dans Diane-Gabrielle Tremblay. Flexibilité, sécurité d'emploi et flexicurité. Les enjeux et les défis. Québec, Presses de l'Université du Québec : 107-132.

FADOQ. (2008). Promotion des droits. <http://www.fadoq.ca/accueil/affichage. asp?langue $=1 \& B=1105>$. Page consultée le 7 octobre 2008.

FADOQ. (2007). Pour favoriser l'adaptation du marché du travail aux changements démographiques. Mémoire présenté par le réseau FADOQ au Groupe d'experts sur les travailleurs âgés. http://www.fadoq.ca/accueil/affichage.asp?langue $=1 \& B=1294$. Page consultée le 7 octobre 2008.

Fourzly, M. et M. Gervais. (2002). Les conventions collectives et les travailleurs âgés au Canada. Développement des ressources humaines Canada. Programme de travail. Canada. http://www1.servicecanada.gc.ca/fra/pt/psait/ctv/pdf/caowc-dftaccc-fr.pdf. Page consultée le 26 septembre 2008.

Fusulier, B., D.-G. Tremblay et M. di Loreto. (2008). « La conciliation emploi-famille dans le secteur du travail social au Québec : une différence de genre ? Quelques éléments de réponse », Les politiques sociales, vol. 3-4:63-81.

Fusulier, B., D-G. Tremblay, T. Moulaert et M. Larivière (2009). Aménagement de la fin de carrière, maintien dans l'emploi et articulation des temps sociaux. Étude comparée du crédit-temps en Belgique francophone (Wallonie-Bruxelles) et des politiques de retraite progressive et de retour à l'emploi au Québec. Rapport de recherche. Coopération Québec-Wallonie-Bruxelles.

Gauthier, Hervé. (dir. 2007). Vie des générations et personnes âgées : aujourd'hui et demain. Volume 2. Québec, Institut de la Statistique du Québec, 403 p. http://www.stat. gouv.qc.ca/publications/conditions/pdf2007/VieGenerationVo2.pdf. Page consultée le 26 septembre 2009.

Gauthier, H., P.-O. Ménard, C. Desfossés et A-M. Fadel. (2007). « Participation au marché du travail, travailleurs âgés et retraite », dans Hervé Gauthier (dir.). Vie des générations et personnes âgées : aujourd'hui et demain. Québec. ISQ : 173-216. http://www.stat.gouv. qc.ca/publications/conditions/pdf2007/generation_vol2_chap10.pdf. Page consultée le 26 septembre 2009.

Gouvernement du Québec. (2004). Projets pilotes à l'intention des travailleurs âgés : sondage auprès des personnes participantes. Rapport d'évaluation. Québec, Direction générale des politiques.http://www.emploi-quebec.net/publications/Liens-indirects/00_ etude_tages_sondagepart.pdf. Page consultée le 26 septembre 2009.

Gouvernement du Québec. (2003). Stratégie d'intervention à l'intention des travailleuses et des travailleurs de 45 ans et plus. Pour que toutes et tous profitent du plein emploi. Québec. http://emploiquebec.net/publications/pdf/00_imt_45ansplus.pdf. Page consultée 


\section{Les défis de fins de carrière et la retraite : le cas du Québec}

le 26 septembre 2009.

Lizée, Michel. (2007). « Incitation à l'allongement de la vie active et retrait progressive, deux outils pour faire face au vieillissement de la main-d'œuvre », Chronique internationale de l'IRES, n¹09, novembre : 127-137.

Malcolm H. Morrison. (1985). The Transition to Retirement. Washington, Bureau of Social Science Research.

Régie des rentes du Québec. (2007). Régime des Rentes du Québec. Statistiques de l'année 2006. Québec, Régie des rentes du Québec.

RHDSC. (2008). Indicateurs de mieux-être au Canada. Sécurité financière - revenu de retraite. Calculs fondés selon les données de Statistique Canada. Caractéristiques des aînés, selon le groupe d'âge et la source de revenu, annuel (tableau CANSIM 111-0035), Ottawa, Statistique Canada. http://www4.rhdsc.gc.ca/.3ndic.1t.4r@-fra.jsp?preview=1\&iid $=27 \& b w=1$. Page consultée le 26 septembre 2009 .

Schellenberg, Grant et Yuri Ostrovsky. (2008). « Rapport de l'Enquête sociale générale de 2007. Planification de retraite et attentes des travailleurs plus âgés », Tendances sociales canadiennes, Statistique Canada, 11-008 : 11-35.

Tremblay, Diane-Gabrielle. (dir. 2007). D'une culture de la retraite à un nouveau management des âges et des temps sociaux. Québec, Presses de l'université du Québec, Collection Économie politique, $281 \mathrm{p}$.

Tremblay, Diane-Gabrielle, Émilie Genin (2009a). Rapport de recherche sur les fins de carrières. Montréal: Chaire de recherche sur les enjeux socio-organisationnels de l'économie du savoir et Alliance de recherche (ARUC) sur la gestion des ages et des temps sociaux. 60 p.

Tremblay, D.-G., É. Genin (2009b). “Aging, economic insecurity and employment: Which measures would encourage older workers to stay longer in the labour market?" A paraître dans Studies in Social Justice, special Issue on Employment and Economic Inequalities. Tremblay, D.-G. et E. Najem (2009). « Work, age and working time preferences : towards a new articulation of social times in a context of economic crisis?" The International Journal for Responsible Employment Practices and Principles.Vol. 1, no 1. Pp. 53-71.

Tremblay, D.-G. (2008a). « The ages of life, work-family balance and aspirations in working time; challenges for firms and countries », Journal of Applied Business and Economics (ISSN\# 1499-691X), vol. 8 (2) : 72-84. http://www.na-businesspress.com/jabeopen.html. Page consultée le 26 septembre 2009.

Tremblay, D.-G. (dir. 2008b). Flexibilité, sécurité d'emploi et flexicurité : les enjeux et défis. Québec, Presses de l'université du Québec, 358 p.

Tremblay, D.-G., E. Najem et R. Paquet. (2008). "Les aspirations des travailleurs vieillissants en matière de fin de carrière : vers de nouvelles temporalités sociales? ", Les Politiques sociales, vol. 3-4 : 76-94. 